\title{
Mechanical Properties of Hydrocolloid and Rubber Impression Materials
}

\author{
G. W. MAC PHERSON, * R. G. CRAIG, and F. A. PEYTON \\ University of Michigan, School of Dentistry, Ann Arbor, Michigan
}

Hampson ${ }^{1}$ has studied the elasticity of alginates under compression and has observed that materials with smaller amounts of permanent deformation were more suitable for use in the presence of undercut areas. Schoonover and Dickson ${ }^{2}$ report the breaking stress, percent strain, and percent set of alginates. Cresson ${ }^{3}$ suggested the revision of the testing procedure for compressive strengths of dental elastic impression materials that would apply the load at the rate of $18,000 \mathrm{Gm}$. per minute. Skinner et al. ${ }^{4}$ and Phillips ${ }^{5}$ point out the importance of the rate of deformation on the technic of handling alginate hydrocolloids. Warner ${ }^{6}$ also shows that the breaking strength and percent deformation of alginates are functions of the loading rate where the higher the rate, the greater is the strength qualities. Anderson ${ }^{7}$ shows the recovery from deformation of alginate to be a direct function of time from the start of mixing. Donnison and Docking 8 have measured the permanent deformation, stiffness, and toughness of alginates, the latter being determined as a combination of compressive strength and the strain at rupture. The ADA Specification $18^{9}$ requires the compressive strength to be determined at a rate of $10,000 \pm 2,000$ $\mathrm{Gm}$. per minute. Based on an average strength of $7,000 \mathrm{Gm} . / \mathrm{cm}^{2}$ and a specimen $1 / 2$ inch in diameter, the time required for rupture would be approximately half a minute.

Recently, Braden ${ }^{10}$ has determined the energy of tearing of one each of mercaptan, silicone, and alginate impression materials as a function of the rate of tearing and the temperature of testing. As the rate of tearing increased and the temperature decreased,

\footnotetext{
This study was supported by the W. K. Kellog Foundation in the form of a fellowship during 1962-63. Received for publication November 15, 1966.

* Present address: School of Dentistry, University of Chile, Santiago, Chile.
}

the energy of tearing increased. The purpose of this investigation was to measure the stress-strain properties in compression, and the resistance to tearing, and the effect of loading rate on these properties. The emphasis will be placed on alginate impression materials, and some values for agar hydrocolloid and mercaptan rubber-base materials will be reported.

\section{Materials and Methods}

The alginate, agar, and mercaptan materials used in the study were assigned code letters (Table 1).

Preparation of test SPECIMENS.-Test specimens for determining the stress-strain curves, compressive strength, permanent deformation caused by a fixed strain or a fixed stress, and the strain in compression were cylinders $3 / 4$ inch long and $1 / 2$ inch in diameter. The specimens of alginate were prepared as described in ADA Specification No. $18 .^{9}$ The specimens usually were separated from the mold at 5 minutes 30 seconds and stored in a humidor at 100 percent relative humidity until tested. When the specimens were tested at 4 minutes from the start of the mix, they were removed from the mold at 3 minutes 45 seconds and tested immediately. The test specimens of mercaptans were prepared according to the proposed specification for rubber-base impression materials. ${ }^{11}$ The rubber specimens were separated from the mold at 7 minutes 30 seconds from the start of mixing and tested at 8 or at 12 minutes. The test specimens of agar impression materials were prepared according to ADA Specification 11 for dental hydrocolloid impression material, agar type. ${ }^{12}$

All tear specimens had the shape of the ASTM tear-die C (ASTM Test D 62454). ${ }^{13}$ The specimens were crescent-shaped with a radius of curvature of $1 / 2$ inch on one 
TABLE 1

IMPREsSion Materials Tested

\begin{tabular}{|c|c|c|c|}
\hline$\overline{\text { Code }}$ & Trade Brand & Batch No. & Manufacturer \\
\hline \multicolumn{4}{|c|}{ Alginates } \\
\hline $\mathrm{A}$ & Jeltrate & 108623 & L. D. Caulk Co., Milford, Del. \\
\hline B & Key to Alginates & 234 & Dental Perfection Co., Inc., Glendale, Calif \\
\hline $\mathrm{C}$ & Coe Alginate & 03052 & Coe Laboratories, Inc., Chicago, Ill. \\
\hline $\mathrm{D}$ & D. P. Elastic Impression Cream & 4815 & Dental Perfection Co., Inc., Glendale, Calif. \\
\hline $\mathbf{E}$ & Inlay Jeltrate & $\begin{array}{l}17262 \\
7460\end{array}$ & $\begin{array}{l}\text { L. D. Caulk Co., Milford, Del. } \\
\text { L. D. Caulk Co., Milford, Del. }\end{array}$ \\
\hline \multicolumn{4}{|c|}{ Rubber Base } \\
\hline $\mathbf{F}$ & Permlastic, Light Bodied & $0524 \mathrm{~A} 205$ & Kerr Manufacturing Co., Detroit, Mich. \\
\hline $\mathrm{G}$ & Permlastic, Heavy Bodied & $0622 \mathrm{~A} 244$ & Kerr Manufacturing Co., Detroit, Mich. \\
\hline $\mathbf{H}$ & Rubberjel, Syringe Type & $059-561$ & L. D. Caulk Co., Milford, Del. \\
\hline I & Rubberjel, Tray Type & $\begin{array}{l}059-502 \\
059-559\end{array}$ & L. D. Caulk Co., Milford, Del. \\
\hline \multicolumn{4}{|l|}{ Agar } \\
\hline J & Surgident, Tray Type & 3615065 & Surgident, Ltd., Los Angeles, Calif. \\
\hline $\mathbf{K}$ & Surgident, Syringe Type & $8-61$ & Surgident, Ltd., Los Angeles, Calif. \\
\hline $\mathrm{L}$ & Deelastic & & Kerr Manufacturing Co., Detroit, Mich. \\
\hline M & $\operatorname{Van} \mathrm{R}$. & 7628 & Walter J. Van Rossem, Los Angeles, Calif. \\
\hline
\end{tabular}

side and a 90-degree notch on the other. The mold was $4 \times 11 / 16 \times 3 / 8$ inches and was prepared in a processed casting plastic. The impression material was poured into the mold placed on a glass plate, and the excess was squeezed out by placing a second glass plate on the top of the mold. The thickness of the specimens was determined with a standard thickness gauge for testing rubber. The normal consistencies were obtained by reducing or increasing the weight of the powder for a normal mix by one-sixth. This decision was based on ten observations for each material of the amounts normally dispensed; it was found the highest and lowest weights varied from the mean by approximately one-sixth.

Testing PRocedures. - The compressive strength of the alginates was determined 6 minutes from the start of the mix, using a tensile-compression instrument.* The rate of loading was $40 \pm 10 \mathrm{lb}$. per minute until fracture, and the maximum load was recorded to the nearest $0.1 \mathrm{lb}$. The compressive strength was determined from the stress-strain curves obtained at a loading rate of 10 inches per minute. $t$ The percent compression at rupture also was determined from these curves.

The permanent deformation from a fixed strain of 12 percent was determined according to ADA Specification $18^{9}$ for some of the

* Riehle Testing Machine, American Machine and Metals, Inc., East Moline, Ill.

$\dagger$ Instron Testing Machine, Instron Engineering Corporation, Quincy, Mass.

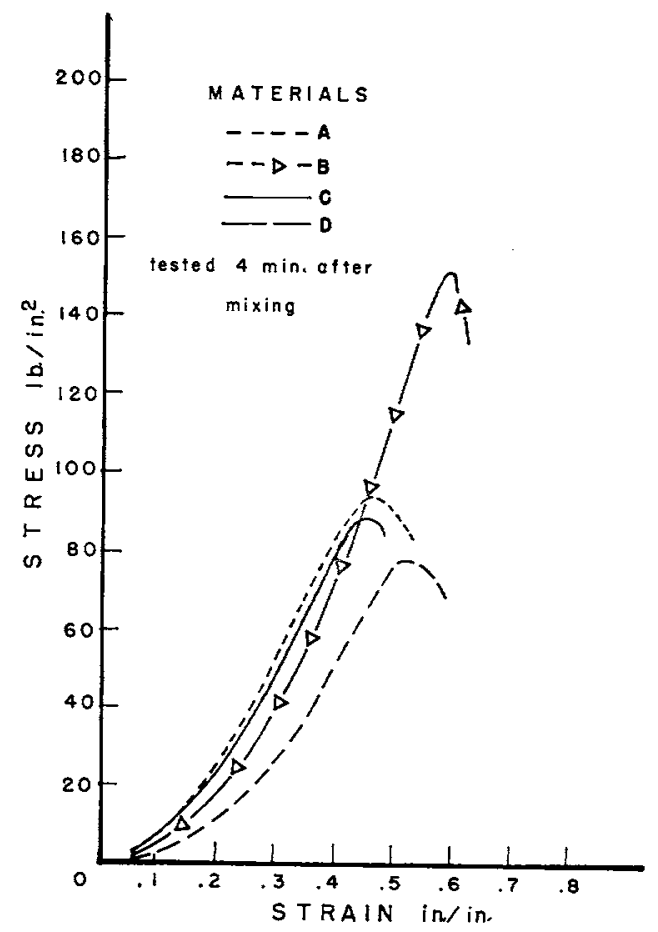

FIG. 1.-Stress-strain curves in compression for alginates $\mathrm{A}, \mathrm{B}, \mathrm{C}$, and $\mathrm{D}$ for 4 minutes from the start of the mix.

specimens. Other samples were strain-tested at 60 rather than 30 seconds, with 60 seconds allowed for recovery rather than 30 seconds. To compare the ADA test with the test used in the Australian Dental Standards, ${ }^{14}$ some 


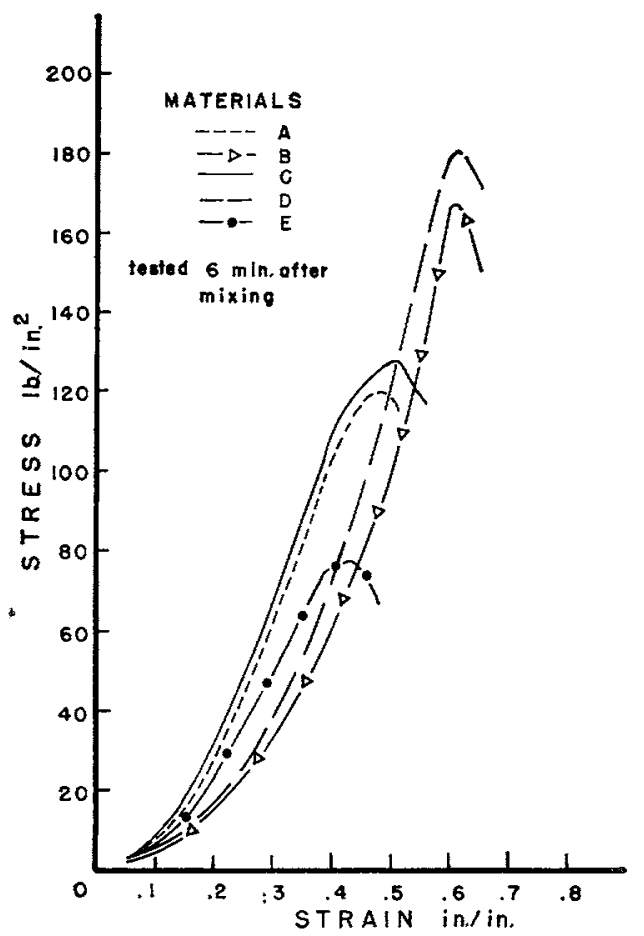

FIG. 2.-Stress-strain curves in compression of alginate gels six minutes from the start of the mix.

samples were tested at 10 percent strain as specified by the latter test.

The strain in compression and permanent deformation from a fixed stress was measured according to ADA Specification $18,{ }^{\circ}$ except the time from the start of the mix to the time of testing was 4,6 , or 10 minutes. In all instances, three samples were used to obtain the means.

\section{Results}

The stress-strain curves in compression for the alginate materials $A, B, C$, and D, determined 4 minutes from the time of mixing, are shown (Fig. 1). Under these test conditions, the entire stress-strain curve was obtained in 2 to 3 seconds. The maximums in the stress-strain curves resulted from the rupture of the alginate being gradual rather than rapid, as in brittle fracture. The curve for material $\mathrm{E}$ was not shown (Fig. 1) because the setting time was 4 minutes 30 seconds. The height of the stress-strain curves measured at 4 minutes was in the same order as the setting times of the alginate impression materials A to D. For purposes of com- parison, the stress-strain curves 6 minutes after mixing are shown (Fig. 2). In the period from 4 to 6 minutes, the order of alginate $D$ changed from the lowest to the highest strength material. The fast-setting material $B$ only slightly improved in strength during this period; alginates $\mathrm{A}$ and $\mathrm{C}$ had modest increases in strength. The stress-strain curve for alginate $\mathrm{E}$ is also shown (Fig. 2); it had the lowest strength, which is characteristic of a syringe material. A further comparison of the stress-strain properties (Fig. 3) shows the curves for mercaptan rubbers $\mathrm{H}$ and $\mathrm{I}, 8$ and $12 \mathrm{~min}$ utes after the start of the mix. The mercaptan samples did not rupture during testing; the specimens simply flattened out as the stress increased. Little improvement was observed in the elastic qualities of tray mercaptan I at 8 to 12 minutes, although considerable improvement occurred with syringe mercaptan $\mathrm{H}$.

The cord modulus of the alginate and mercaptan materials at 5 percent strain and the maximum percent compression of the

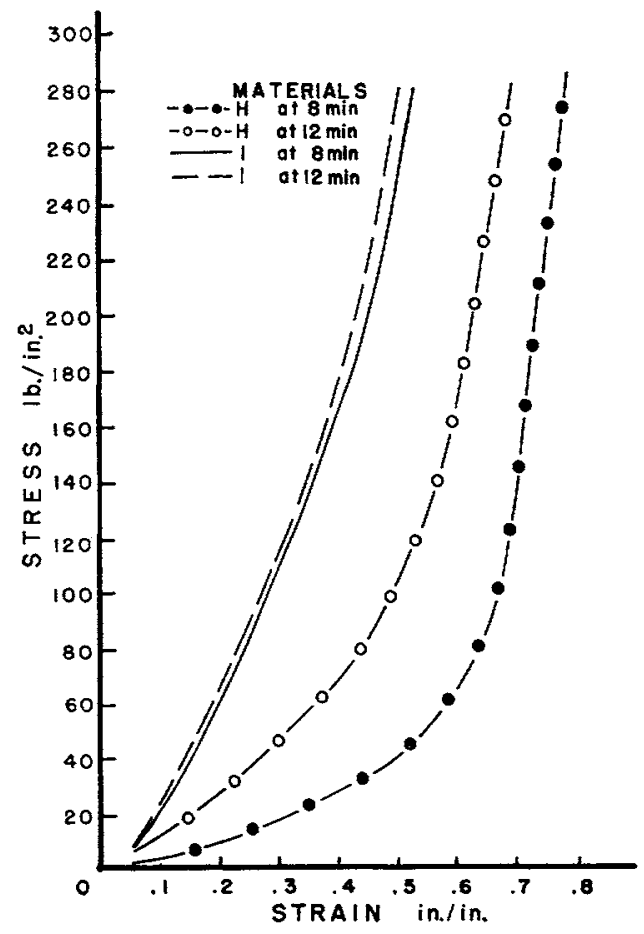

FIG. 3.-Stress-strain curves in compression of mercaptan $H$ and $I$ at eight and twelve minutes from the start of the mix. 
TABLE 2

Stress-Strain Properties in

Compression of Alginates* and Mercaptans $\dagger$

\begin{tabular}{ccc}
\hline \hline & $\begin{array}{c}\text { Cord Modulus } \\
\text { At } 5 \text { Percent Strain } \\
\text { (1b./in. }{ }^{2} \text { ) }\end{array}$ & $\begin{array}{c}\text { Maximum Compression } \\
\text { (Percent) }\end{array}$ \\
\hline A & 38 & 48 \\
B & 28 & 67 \\
C & 47 & 51 \\
D & 28 & 61 \\
E & 38 & 43 \\
H & 28 & $\cdots$ \\
I & 105 & $\cdots$ \\
\hline
\end{tabular}

*At 6 minutes from mixing.

†At 8 minutes from mixing.

alginates are listed (Table 2). The order was not the same for the cord moduli as for the maximum compressive strengths. Thus, at low deformation, $\mathrm{A}, \mathrm{C}$, and $\mathrm{E}$ were significantly stiffer than alginates $\mathrm{B}$ and $\mathrm{D}$ and mercaptan H. Mercaptan I, 8 minutes from the start of the mix, was the stiffest material tested. The percent compression of the alginates prior to rupture were in the inverse order of the stiffness, as indicated by the cord moduli.

The compressive strengths of the alginates are listed (Table 3 ). The strengths at normal consistency were calculated from the stress-strain curves at the more rapid rate of 10 inches per minute. The latter strengths were consistently higher than those obtained with a rate of loading of $40 \mathrm{lb}$. per minute, and the difference in the means were significant at the 99 percent confidence level, based on the $t$ test. Thin and thick consistency of mixes resulted in lower and higher compressive strengths than normal mixes of corresponding materials; the differences were significant at the 95 percent

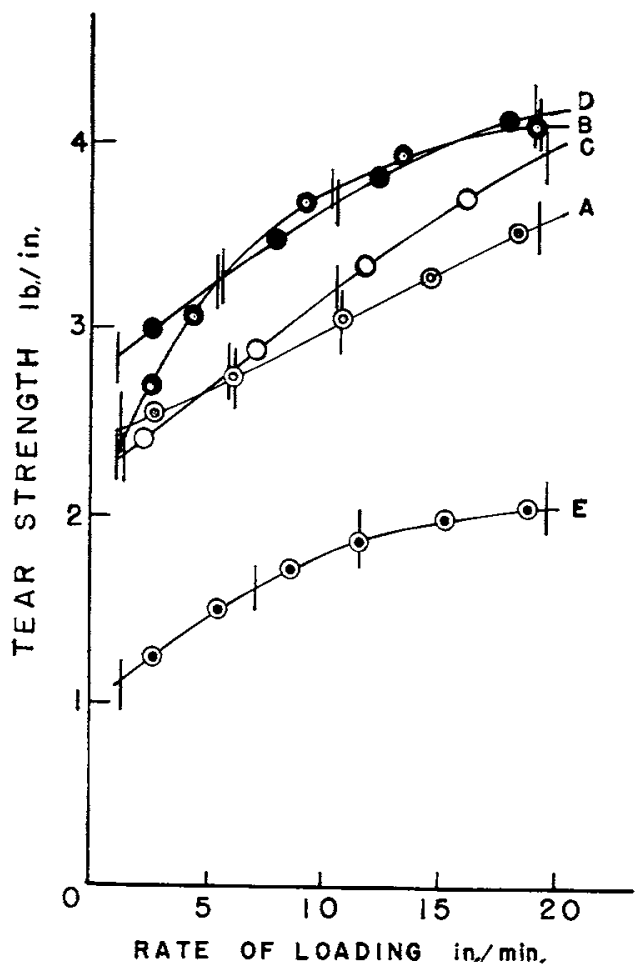

FIG. 4.-The effect of the rate of loading on the tear resistance of alginate impression materials.

level of confidence, using a two-way analysis of variance and an $F_{2,6}$ ratio of 24.9. A comparison of the materials $\mathrm{A}$ to $\mathrm{E}$ at normal, thin, and thick consistencies, using an analysis of variance, showed the compressive strengths were significantly different at the 95 percent confidence level based on an $\mathrm{F}_{4,10}$ ratio of 138.4 .

The influence of rate of loading on the resistance to tearing of the alginates mixed

TABLE 3

EfFect of the CONSISTENCY ON THE

Compressive Strength of Alginate Impression Materials

\begin{tabular}{|c|c|c|c|c|c|c|c|c|}
\hline \multirow[b]{3}{*}{ Code } & \multicolumn{8}{|c|}{ Compressive Strength } \\
\hline & \multicolumn{4}{|c|}{ Normal Consistency } & \multicolumn{2}{|c|}{ Thin Consistency } & \multicolumn{2}{|c|}{ Thick Consistency } \\
\hline & $\mathrm{Gm} . / \mathrm{cm}^{2}$ & s.d. & $\mathrm{Gm} . / \mathrm{cm}^{2}$ & s.d. & $\mathrm{Gm} \cdot / \mathrm{cm}^{2}$ & s.d. & $\mathrm{Gm} . / \mathrm{cm}^{2}$ & s.d. \\
\hline A & 6,200 & 440 & $8,400^{*}$ & 180 & 5,300 & 260 & 7,200 & 230 \\
\hline B & 5,300 & 260 & 11,700 & 200 & 4,300 & 200 & 6,800 & 100 \\
\hline C & 6,900 & 000 & 8,900 & 220 & 5,600 & 180 & 8,400 & 100 \\
\hline D & 8,000 & 410 & 12,600 & 310 & 6,400 & 270 & 9,400 & 20 \\
\hline $\mathbf{E}$ & 2,200 & 320 & 5,400 & 180 & 1,800 & 60 & 2,600 & 130 \\
\hline
\end{tabular}

*Loading rate 10 inches per minute. 


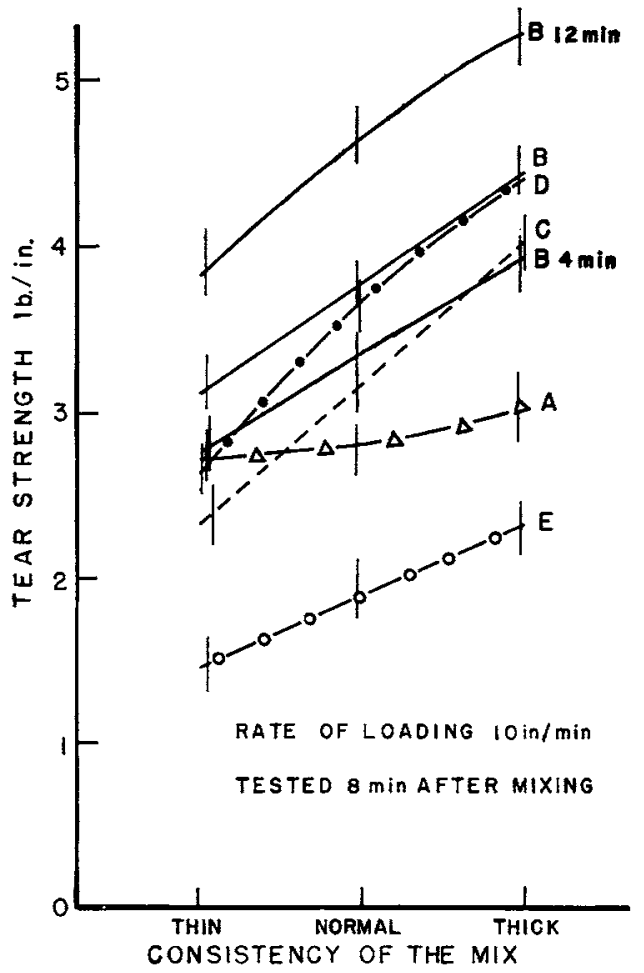

FIG. 5.-The effect of consistency and time of testing on the resistance to tearing of alginate impression materials.

at normal consistency is illustrated (Fig. 4) with vertical lines representing the range of results. All materials showed an increase in tear strength with an increase in the rate of loading, and an analysis of variance showed that the increase was significant at the 95 percent confidence level.* Ranking, using Duncan's multiple range test, showed alginates $\mathrm{B}$ and $\mathrm{D}$ and alginates $\mathrm{A}$ and $\mathrm{C}$ were in the same groups, and $\mathrm{E}$ was distinct from each of these groups.

The effect of altering the consistency on the tear strength is shown (Fig. 5) with the rate of loading held constant at 10 inches per minute. An analysis of variance showed the alginates had higher tear strengths the stiffer the consistencies. The rate of increase of tear strength was approximately the same for the alginates, with the exception of alginate $A$, which had only a slight increase in tear strength from thin to thick consistency. For alginate $B$, the tear strengths also are shown for 4 and 12 minutes after mixing.

* All further reference to significance will be at the 95 percent level.

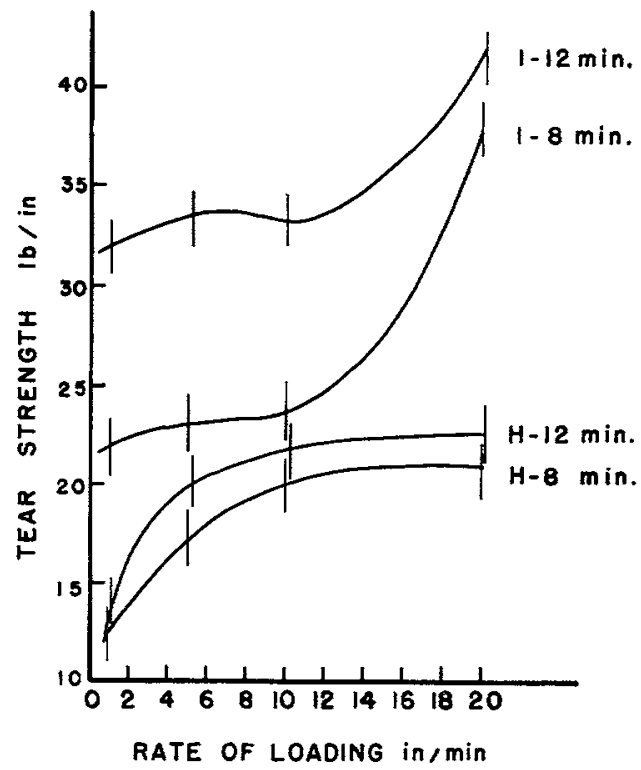

FIG. 6.-The effect of rate of loading on the tear resistance of mercaptan materials $H$ and $I$.

An analysis of variance showed that an increase in time of testing between 4 and 12 minutes resulted in increase in tear strengths.

The tear strengths of mercaptan and agar impression materials are shown (Fig. 6; Table 4). The mercaptan tray and syringe materials, $\mathrm{I}$ and $\mathrm{H}$, possessed higher tear strengths, and the values at 8 minutes for the agar were comparable to those of the alginate materials. Increased rates of loading increased the tear strengths of the mercaptans; however, the tear strengths, even at low rates, were higher than the values of the hydrocolloids. The tear strength of tray material I increased substantially with time, but the syringe material $\mathrm{H}$ did not show any distinct improvement.

TABLE 4

Effect of the Time of Testing from the Setting Time on the Tear Strength of Agar HydrocolloIds*

\begin{tabular}{llllll}
\hline \hline & \multicolumn{2}{c}{5 Minutes } & & \multicolumn{2}{c}{8 Minutes } \\
\cline { 2 - 3 } \cline { 5 - 6 } Code & lb./in. & s.d. & & lb./in. & s.d. \\
\hline J & 4.79 & 0.44 & & 5.68 & 0.08 \\
K & 4.00 & 0.30 & & $\ldots .51$ & 0.12 \\
L & 1.69 & 0.37 & & 2.51 & 0.12 \\
M & 2.95 & 0.70 & & 3.39 & 0.45 \\
\hline
\end{tabular}

* Rate of testing 10 inches per minute. 
TABLE 5

EFfect of the CONSISTENCY OF THE Mix ON the Permanent Deformation Caused by

Fixed Strain of

Alginate IMPREssion Materials*

\begin{tabular}{|c|c|c|c|c|c|c|}
\hline \multirow[b]{3}{*}{ Code } & \multicolumn{6}{|c|}{ Percent Permanent Deformation } \\
\hline & \multicolumn{2}{|c|}{$\begin{array}{l}\text { Normal } \\
\text { Consistency }\end{array}$} & \multicolumn{2}{|c|}{$\begin{array}{l}\text { Thin } \\
\text { Consistency }\end{array}$} & \multicolumn{2}{|c|}{$\begin{array}{c}\text { Thick } \\
\text { Consistency }\end{array}$} \\
\hline & Mean & s.d. & Mean & s.d. & Mean & s.d. \\
\hline A & 2.2 & 0.1 & 2.2 & 0.1 & 2.1 & 0.1 \\
\hline $\mathrm{B}$ & 2.2 & 0.3 & 2.3 & 0.2 & 2.6 & 0.1 \\
\hline $\mathrm{C}$ & 2.5 & 0.1 & 2.6 & 0.2 & 2.5 & 0.1 \\
\hline $\mathrm{D}$ & 2.1 & 0.2 & 2.0 & 0.2 & 2.0 & 0.1 \\
\hline $\mathrm{E}$ & 2.5 & $0 . \overline{1}$ & 2.6 & 0.1 & 2.6 & 0.1 \\
\hline
\end{tabular}

*12 percent strain for 1 minute. Measured 8 minutes after the start of the mix.

The influence of the consistency and time of testing on the permanent deformation of a fixed strain of 12 percent is presented (Tables 5,6 ). An analysis of variance of these data showed that the normal variation in consistency of the mixes and the time of testing between 4 and 10 minutes had no significant influence on the permanent deformation. Also, no significant difference was observed between these values and the permanent deformation obtained when a 10 percent strain was used, as advocated by the Australian Dental Specification. For example, 2.4 and 1.6 percent permanent deformation was obtained with alginate $C$ at 4 and 6 minutes when a value of 10 percent strain was used in the test.

The effect of the consistency and time of testing on the strain in compression are given (Table 7 ). The change in consistency from thin to thick resulted in a significant decrease in strain in compression, based on an $F_{2,6}$ ratio of 130 . A greater increase in stiffness resulted from changing the con-
TABLE 6

Permanent Deformation at Fixed Strain of Alginate Impression Materials*

\begin{tabular}{|c|c|c|c|c|c|c|}
\hline \multirow[b]{3}{*}{ Code } & \multicolumn{6}{|c|}{ Percent Permanent Deformation } \\
\hline & \multicolumn{2}{|c|}{4 Minutes } & \multicolumn{2}{|c|}{6 Minutes } & \multicolumn{2}{|c|}{10 Minutes } \\
\hline & Mean & s.d. & Mean & s.d. & Mean & s.d. \\
\hline A & 2.4 & 0.1 & 2.2 & 0.1 & 2.0 & 0.1 \\
\hline $\mathrm{B}$ & 2.5 & 0.1 & 2.3 & 0.1 & 1.9 & 0.2 \\
\hline $\mathrm{C}$ & 2.4 & 0.1 & 1.9 & 0.1 & 1.6 & 0.1 \\
\hline $\mathrm{D}$ & 2.7 & 0.4 & 2.1 & 0.1 & 1.8 & 0.1 \\
\hline $\mathbf{E}$ & 2.5 & 0.1 & 2.1 & 0.1 & 1.3 & 0.1 \\
\hline
\end{tabular}

*12 percent strain for 30 seconds; normal consistency.

sistency from thin to normal than from normal to thick. No significant difference was observed when the strain in compression was measured at 4,6 , and 10 minutes from the start of the mix $\left(\mathrm{F}_{2,6}=0.73\right)$. The permanent deformation observed after loading with a constant stress is reported (Table 8 ). No significant difference was found in the percent permanent deformation when the time of testing was increased from 4 to 10 minutes $\left(\mathrm{F}_{2,6}=0.14\right)$. Increasing the consistency from thin to thick did have a significant influence on decreasing the permanent deformation of the alginates $\left(\mathrm{F}_{2,6}\right.$ $=45.6$ ).

In comparing the strain in compression and permanent deformation caused by a fixed stress of mercaptans (Table 9), the strain in compression was lower for the mercaptans than the alginates, and the tray materials had approximately half the strain of the corresponding syringe materials. The permanent deformation of one mercaptan type, $\mathrm{F}$-syringe and $\mathrm{G}$-tray, was comparable to the alginates; however, the second mercaptan type, H-syringe and I-tray, had significantly less permanent deformation.

TABLE 7

EFfect of Consistency and Time of Testing

on the Strain in Compression of Alginate Impression Materials

\begin{tabular}{|c|c|c|c|c|c|c|c|c|c|c|}
\hline \multirow[b]{3}{*}{ Code } & \multicolumn{10}{|c|}{ Percent Strain } \\
\hline & \multicolumn{6}{|c|}{ Normal Consistency } & \multicolumn{2}{|c|}{ Thin Consistency } & \multicolumn{2}{|c|}{ Thick Consistency } \\
\hline & 4 Minutes & s.d. & 6 Minutes & s.d. & 10 Minutes & s.d. & 6 Minutes & s.d. & 6 Minutes & s.d. \\
\hline A & 13.0 & 0.2 & 13.4 & 0.7 & 13.1 & 0.2 & 18.5 & 0.9 & 10.1 & 0.3 \\
\hline B & 18.1 & 0.4 & 19.4 & 0.4 & 18.7 & 0.4 & 23.1 & 0.4 & 18.3 & 0.2 \\
\hline C & 13.3 & 0.2 & 13.1 & 0.1 & 13.3 & 0.3 & 16.2 & 1.4 & 12.5 & 0.4 \\
\hline $\mathrm{D}$ & 16.9 & 0.4 & 17.1 & 0.7 & 16.5 & 0.2 & 19.6 & 0.2 & 16.3 & 0.2 \\
\hline $\mathrm{E}$ & 12.9 & 0.3 & 12.9 & 0.2 & 13.1 & 0.5 & 14.8 & 0.5 & 10.9 & 0.4 \\
\hline
\end{tabular}


TABLE 8

Effect of Consistency and Time of Testing on the Permanent Deformation Caused by a Fixed Stress of Alginate Impression Materials

\begin{tabular}{|c|c|c|c|c|c|c|c|c|c|c|}
\hline \multirow[b]{3}{*}{ Code } & \multicolumn{10}{|c|}{ Percent Permanent Deformation } \\
\hline & \multicolumn{6}{|c|}{ Normal Consistency } & \multicolumn{2}{|c|}{ Thin Consistency } & \multicolumn{2}{|c|}{ Thick Consistency } \\
\hline & 4 Minutes & s.d. & 6 Minutes & s.d. & 10 Minutes & s.d. & 6 Minutes & s.d. & 6 Minutes & s.d. \\
\hline A & 3.6 & 0.1 & 3.6 & 0.1 & 3.6 & 0.1 & 6.3 & 0.8 & 2.7 & 0.2 \\
\hline B & 6.5 & 0.2 & 6.5 & 0.3 & 5.9 & 0.2 & 8.7 & 0.5 & 5.4 & 0.1 \\
\hline $\mathrm{C}$ & 4.0 & 0.1 & 3.4 & 0.6 & 3.0 & 0.1 & 4.0 & 0.1 & 2.8 & 0.1 \\
\hline $\mathrm{D}$ & 4.8 & 1.0 & 4.0 & 0.1 & 4.2 & 0.3 & 4.9 & 0.1 & 3.6 & 0.1 \\
\hline $\mathrm{E}$ & 3.9 & 0.4 & 3.4 & 0.2 & 3.7 & 0.3 & 4.5 & 0.2 & 2.7 & 0.2 \\
\hline
\end{tabular}

TABLE 9

Strain in Compression ano Permanent Deformation Caused by a Fixed Stress of RubBer-Base Impression Materials*

\begin{tabular}{|c|c|c|c|c|}
\hline Code & $\begin{array}{l}\text { Percent } \\
\text { Strain }\end{array}$ & s.d. & $\begin{array}{c}\text { Percent } \\
\text { Permanent } \\
\text { Deformation }\end{array}$ & s.d. \\
\hline $\mathrm{F}$ & 11.5 & 0.4 & 4.1 & 0.2 \\
\hline $\mathrm{G}$ & 6.5 & 0.2 & 3.7 & 0.1 \\
\hline $\mathrm{H}$ & 9.5 & 0.3 & 1.6 & 0.1 \\
\hline $\mathrm{I}$ & 5.1 & 0.2 & 0.5 & 0.1 \\
\hline
\end{tabular}

*At 8 minutes.

\section{Discussion}

The stress-strain, strength, percent permanent deformation at constant strain of constant stress, and the percent strain at a constant stress of elastic impression materials are important in clinical applications of these products. Of foremost importance is the general observation that the elastic and plastic properties of alginate, agar, and mercaptan rubber impression materials are a function of: (1) the rate at which the materials are deformed, (2) the time at which the material is tested, and (3) the consistency of the mix.

It is important to understand that the compressive and tear strengths of the alginates increase as the rate of load application is increased. These materials, therefore, function as more nearly elastic materials the more rapidly they are deformed. This is particularily important when thin sections are involved in the impression, since equivalent forces or deformation result in higher corresponding stresses and strains than in thick sections. Increasing the bulk between the tray and impression surface will reduce the strain during removal over undercut areas. Other means of improving the chance of obtaining a good alginate impression are (1) to increase the consistency of the $\mathrm{mix}$ within clinical limitations, (2) to allow a few minutes extra before removing the impression, or (3) to increase the rate of deformation during the removal of impressions. These methods increase the elastic and decrease the plastic qualities of the alginate gels.

The measurement of strain (flexibility) and permanent deformation at low constant stresses compared to that required for mechanical failure of the gels, has been shown to be a function more of the consistency of the mix than a function of time of testing from 4 to 10 minutes from the start of the mixing. The percent permanent deformation at constant strain of low magnitudes (10 to 12 percent) was not affected by the time from the start of the mix to the time of testing or the alterations in consistency from thin to thick. It is apparent that the test at constant strain is a less severe test of the elastic qualities than that run at a constant stress where more plastic deformation resulted. It is concluded, therefore, that the permanent deformation, or plastic quality of the gels, is minimized by maintaining the consistency of the mixes of alginates as thick as is clinically feasible.

\section{Summary}

The elastic and plastic qualities of alginate gels were determined by measuring the resistance to tearing and the stress-strain properties in compression. The tear strength and compressive strengths were determined as a function of alginate concentration, the time of testing, and the rate of load application. The strain at constant stress as well as the permanent deformation at constant 
stress and constant strain were determined.

The tear strengths and the compressive strengths were shown to increase with (1) an increase in rate of loading, (2) an increase in the amount of alginate in the mix, and (3) an increase in the time between mixing and testing. The resistance to tearing of the alginates was comparable to agar impression materials but was much lower than those of two mercaptan products.

The strain and permanent deformation measured at a constant stress was decreased when more alginate was used. Variation in the time of testing from 4 to 10 minutes had no significant influence on the strain or permanent deformation measured at constant stress.

The permanent deformation at a fixed strain of 10 to 12 percent was significantly influenced by the change in alginate concentration studied but was not significantly affected by the variation of 4 to 10 minutes in the time of testing.

\section{References}

1. Hampson, E. L. Some Observations on the Effect of Compression on Impression Materials Which Are Made from Reversible and Irreversible Hydrocolloids and Rubber-base Materials, Dent. Practit. $\mathcal{E}$ dent. Rec., 6:250-55, 1956.

2. Schoonover, I. C., and Dickson, G. Preparation and Characteristics of Elastic Dental Impression
Compounds With an Alginate Base, J. Amer. dent. Assn., 30:565-69, 1943 .

3. Cresson, J. Suggested Revision for Testing Dental Elastic Impression Materials, J. dent. Res., 28:573, 1949. Abstract.

4. Skinner, E. W., CoOper, E. N., and Beck, F. E. Reversible and Irreversible Hydrocolloid Impression Materials, J. Amer. dent. Assn., 40:196-207, 1950.

5. Phillips, R. W. Physical Properties and Manipulation of Reversible and Irreversible Hydrocolloids, J. Amer. dent. Assn., $51: 566-72,1955$.

6. Warner, H. K. Impression Materials With an Alginate Base, Aust. J. Dent., 48:49-53, 1944.

7. Anderson, J. N. Flow and Elasticity in Alginates, Dent. Progr., I:63-70, 1960

8. Donnison, J. A., and Docking, A. R. Alginate Impression Materials, Aust. dent. J., 5:280-1, 1960.

9. Council on Dental Research, American Dental Association Specification No. 18 for Dental Hydrocolloidal Impression Materials-Alginate Type, $\boldsymbol{l}$. Amer. dent. Assn., 67:758-64, 1963.

10. Braden, M. Characterization of the Rupture Properties of Impression Materials, Dent. Practit. $\mathcal{O}$ dent. Rec., 14:67-71, 1963 .

11. Miller, W. A. C., Jr., Hansen, W. C., Dickson, G., and Sweeney, W. T. Proposed Specification for Impression Material: Synthetic Rubber Base, Dental, J. Amer. dent. Assn., 60:224-29, 1960.

12. Specification No. 11 for Dental Hydrocolloidal Impression Material-Agar Type. In Guide to Dental Materials, ed. 3. Chicago, American Dental Association, 1966 , p. 138-42.

13. Tear Resistance of Vulcanized Rubber, D 624-54. In ASTM Standards 1961, Part II. Philadelphia, American, Society for Testing Materials, 1962, p. $277-79$.

14. Australian Dental Standards No. T 15-1959, Alginate Impression Material. Sydney, Standards Association of Australia. 